\title{
Análise dos parâmetros da qualidade da água destinada a irrigação.
}

Analysis of water quality parameters intended for irrigation.

Análisis de parámetros de calidad del agua destinada para el riego.

Ana Cláudia Marassá Roza Boso

Mestranda, UNESP, Brasil.

Claudia_boso@hotmail

Camila Pires Cremasco Gabriel

Professora Doutora, UNESP, Brasil. camila@tupa.unesp.br

Jhonatan Cabrera Piazentin

Graduando em Licenciatura em Matemática jhonatan_g8@hotmail.com 


\section{RESUMO}

A qualidade da água está relacionada com suas características físicas e destino de uso, sendo de estrema importação a avaliação constante desse recurso tão importante na conservação da vida. Para fins de irrigação, a qualidade da água se destina nos efeitos danosos causados nas peculiaridades da planta cultivada e do solo da plantação. Esses efeitos são estabelecidos de acordo com a concentração da salinidade da água, a permeabilidade do solo e a toxidez da planta. Desta forma, verifica-se a importância de estudos relacionados com indicadores que avaliam da qualidade da água, principalmente em regiões com pouca concentra de tal recurso, permitindo assim o reconhecimento do melhor método a ser utilizado para minimizar futuros problemas e orientar pequenos e grandes produtores. Neste sentido, o vigente trabalho tem por objetivo realizar uma investigação cientifica referente aos cálculos e técnicas utilizadas na avaliação da água no processo de irrigação e seus possíveis danos na qualidade da produção de culturas quanto aos níveis de concentração de cada parâmetro.

PALAVRAS-CHAVE: Indicadores de qualidade. Irrigação. Água.

\section{SUMMARY}

The water quality is related to its physical characteristics and destination of use, being of extreme import the constant evaluation of this very important resource in the preservation of life. For irrigation, water quality intended to harmful effects on the peculiarities of the cultivated plant and planting soil. These effects are determined in accordance with the concentration of water salinity, soil permeability and the toxicity of the plant. Thus, there is the importance of studies related to indicators that assess water quality, especially in regions with little focus of this resource, thereby allowing the recognition of the best method to use to minimize future problems and guide small and large producers. In this sense, the current study aims to conduct a scientific research regarding the calculations and techniques used in the evaluation of water in the irrigation process and possible damage to the quality of crop production as the concentration levels of each parameter.

KEYWORDS: Quality indicators. Irrigation. Water.

\section{RESUMEN}

La calidad del agua se relaciona con sus características físicas y de destino de uso, siendo de importancia extrema la evaluación constante de este recurso tan importante en la preservación de la vida. Para el riego, la calidad del agua destinada a los efectos nocivos sobre las peculiaridades del suelo y la siembra de plantas cultivadas. Estos efectos se determinan de acuerdo con la concentración de la salinidad del agua, la permeabilidad del suelo y la toxicidad de la planta. Por lo tanto, existe la importancia de los estudios relacionados con los indicadores que evalúan la calidad del agua, especialmente en regiones con poco enfoque de este recurso, lo que permite el reconocimiento del mejor método a utilizar para minimizar problemas en el futuro y guiar a los pequeños y grandes productores. En este sentido, el presente estudio tiene por objeto llevar a cabo una investigación científica con respecto a los cálculos y las técnicas utilizadas en la evaluación de agua en el proceso de riego y posibles daños a la calidad de la producción de cultivos como los niveles de concentración de cada parámetro.

PALABRAS CLAVE: indicadores de calidad. Irrigación. Agua. 


\section{INTRODUÇÃO}

Hoje em dia, a água é um recurso essencial em nossas vidas. De maneira geral a mesma é de suma importância para as áreas da saúde, da alimentação, da irrigação, do meio ambiente dentre outros setores. Sua qualidade se define de acordo com suas propriedades biológicas, com o estado que se encontra e com seus efeitos gerados aos usuários (Engman \& Gurney, 1991).

Devido o aumento na produção no setor agrícola, há uma necessidade de avaliações constantes dos recursos hídricos utilizados. Sua qualidade está direcionada com os efeitos danosos aos cultivos e ao solo, necessitando de técnicas especiais para monitorar ou suprir inesperadas contrariedades quanto ao seu aproveitamento (AYERS, R. S.; WESTCOT, D. W.,1991). Sobre o mesmo autor, a autenticidade da água e seus efeitos mencionados, estão relacionados com a concentração da salinidade da água, permeabilidade do solo e a toxidez das plantas do cultivo.

Segundo Almeida (2010), a classificação do uso da água destinado à irrigação segue os seguintes parâmetros:

1-Características químicas, que relaciona a qualidade da água com os componentes químicos e o grau dos efeitos causados indiretamente ou diretamente sobre os cultivos. 2-Caracteristicas agronômicas, que verifica, após analises das características químicas em laboratórios, os danos relacionados com a condutividade elétrica, reduzindo assim os rendimentos da colheita.

3-Caracteristicas edafológicas que afetam a qualidade do cultivo conforme o percentual de sais encontrados na água. Essa concentração pode estar presente na superfície do solo e no desenvolvimento do radicular da planta.

Dentre as características mencionadas, o nível da salinidade é um parâmetro limitativo no progresso de determinadas culturas. Seus efeitos estão relacionados com as caraterísticas do solo, ocasionado assim alterações na estrutura, na permeabilidade e arejo do solo, danificando a evolução das plantas.

São poucas as plantas que utilizam certa quantidade relevante de sais, o que acarreta o crescimento do teor dessa característica no solo por meio de irrigações sucessivas. Entretanto, não se podem definir limites fixos da concentração de salinas permitidas para a água destinada a irrigação, pois há alterações na tolerância de salinidade entre espécies plantas (SILVA, I. N. et al.,2011).

Neste sentido, reconhece-se a importância da constante avaliação da qualidade dos recursos hídricos utilizados na irrigação, principalmente em regiões com baixos índices de chuva, pois essa condição permite a salinização das plantas e sodificação do solo, ou seja, o acumulo de sódio e sais, na raiz das plantas e nos solos utilizados, trazendo grandes prejuízos ao produtor (CORDEIRO, G. G., 2001).

\section{METODOLOGIA}

Este trabalho componha-se de uma revisão literária especializada, no qual se realizou consultas a livros, teses e artigos científicos eleitos por meio de busca eletrônica. A busca foi 
realizada no intuito de analisar e observar quais paramentos utilizados na avaliar da qualidade dá água no meio agrícola. As palavras utilizadas para busca foram qualidade da água e irrigação. Os critérios de incorporação para os estudos são as consequências negativas geradas na produção da cultura e no solo utilizado, devido à concentração das substâncias encontradas na água, e estudos comparativos entre as literaturas selecionadas para o presente trabalho. Foram omitidos estudos que não descreviam tais causas e efeitos e não demostravam os recursos utilizados para avaliação da qualidade. Em seguida, procurou-se estudar, analisar e compreender quais os principais indicadores que são aplicados e empregados nos estudos da qualidade da água destinados a irrigação, de acordo com os índices estabelecidos para tal finalidade.

\section{RESULTADOS}

No contexto geral, por meio de parâmetros que avaliam os níveis de impurezas que são de origens antrópicas ou naturais, a qualidade da água está relacionada com suas características físicas, biológicas e químicas. No entanto, os padrões que definem as características dos recursos hídricos, destinados à irrigação, estão relacionados com a sodicidade, a salinidade, a toxidade e outros (CORDEIRO, G. G. 2001).

\subsection{Salinidade}

A salinidade verifica a concentração de sais no solo, por meio dos recursos hídricos utilizados na irrigação (problema externo). Sua verificação pode ser obtida de duas maneiras. A primeira verifica a concentração da condutividade elétrica $(C E)$ encontrada na água (CORDEIRO, G. G. 2001).

Conforme a Tabela 2, o teor de salinidade do recurso hídrico é expresso pela unidade do Sistema Internacional $(\mathrm{SI})$ e definidas a $25^{\circ} \mathrm{C}$, pois é nessa temperatura que se apresenta a facilidade da corrente atravessar solução e assim obter o resultado desejado (ALMEIDA, O. A., 2010).

Tabela 2: Valores referentes à salinidade da água em seus respectivos recursos.

\begin{tabular}{lr}
\hline FONTE & $\mathrm{CE}^{\mathrm{a} 25^{\circ} \mathrm{C}}$ \\
\hline Água da chuva & $0,15 \mathrm{dSm}^{-1}$ \\
Água média dos rios & $0,2-0,4 \mathrm{dSm}^{-1}$ \\
Água do mar Mediterrâneo & $63 \mathrm{dSm}^{-1}$ \\
Água do oceano Atlântico (máximo) & $59 \mathrm{dSm}^{-1}$ \\
Água do oceano Atlântico (médio) & $55 \mathrm{dSm}^{-1}$ \\
Água do oceano Pacífico & $51 \mathrm{dSm}^{-1}$ \\
Água do oceano Índico & $54 \mathrm{dSm}^{-1}$ \\
Água de irrigação de salinidade média & $0,75-2,25 \mathrm{dSm}^{-1}$ \\
\hline
\end{tabular}

Fonte: ALMEIDA, 2010. 
Assim o parâmetro CE pode ser obtido por meio da expressão:

$$
C E\left(d S m^{-1}\right)=\frac{p p m}{640}=\frac{m e q L^{-1}}{10}=\frac{P o(\text { bar })}{-0,36}
$$

com:

ppm = partes por milhão;

meq $L^{-1}=$ miliequivalentes por litros;

Po $($ bar $)=$ potencial osmático.

Outra maneira de determinar a salinidade da água está na quantidade do TSD (Total de Sais Dissolvidos). Seu valor é definido pela soma dos resultados das concentrações de íons analisados na amostra e expressos por $\mathrm{mg} \mathrm{L}^{-1}$ ou g L ${ }^{-1}$ (CORDEIRO, G.G., 2001).

\subsection{Sodicidade}

A sodicidade é concentração de sódio na água e tem como consequência a toxidade da planta e a permeabilidade do solo sobre a absorção de nutrientes. Sua porcentagem se define pela expressão (AYERS, R. S.; WESTCOT, D., 1991):

$$
\text { Porcentagemdo Sódio }(P S T)=\% N a=\frac{100 * N a^{+}}{\sum \text { cátion }}
$$

No entanto este parâmetro foi substituído por outro parâmetro desenvolvido pelos USA, o RAS(Relação de Adsorção de Sódio). Esse novo parâmetro utilizava uma relação do $\mathrm{Na}^{+}$com o $\mathrm{Mg}^{2}$ e o $\mathrm{Ca}^{2+}$.

$$
R A S=\frac{N a^{+}}{\sqrt{C a^{++}+M g^{++}}}\left(m e q L^{-1}\right)^{1 / 2}=\frac{N a^{+}}{\sqrt{\frac{C a^{++}+M g^{++}}{2}}}\left(m_{m o l} L^{-1}\right)^{1 / 2}
$$

Ao se realizar o $R A S$, não se levou em conta o estado em que se encontrava o solo, fase líquida ou solida. Neste sentido, houve a necessidade de ajuste na relação dos cálculos efetuados pelo $R A S$. Assim obteve-se o $R A S_{a j}$ (Relação de adsorção de sódio ajustada), que avaliavam a concentração do bicarbonato ou carbonato de íons bivalentes presentes na água. Sua expressão é(AYERS, R. S.; WESTCOT, D., 1991):

$$
R A S_{a j}=R A S(1+I S)=R A S[1+(8,4-p H c)]=\frac{N a^{+}}{\sqrt{\frac{c a^{++}+M g^{++}}{2}}}[1+(8,4-p H c)]
$$

Com pHc da seguinte maneira:

$$
p H c=(p K-p K c)+p(C a+M g)+p A l c
$$


De acordo com Suarez (1981) um novo parâmetro foi desenvolvido baseado nos cálculos de $R A S$, o $C E$ da água de irrigação, que define o acumulo de cálcio na água. Ela avalia as modificações feitas pela concentração de cálcio causada no solo. Sua expressão se define na forma:

$$
R A S^{\circ} \frac{N a^{+}}{\sqrt{\frac{C a^{\circ}+M g^{++}}{2}}}
$$

com $\mathrm{Ca}^{\circ}$ definida pela ocorrência de cálcio corrigido.

Contudo, a forma mais utilizada nos meio agrícolas para análise da água é o $R A S^{\circ}$, pois os seus resultados se equivalem aos obtidos pelo $R A S$ e o $R A S_{a j}$ não verificavam com clareja os perigos da sodicidade.

Figura 1: Classificação da água em relação à socididade.

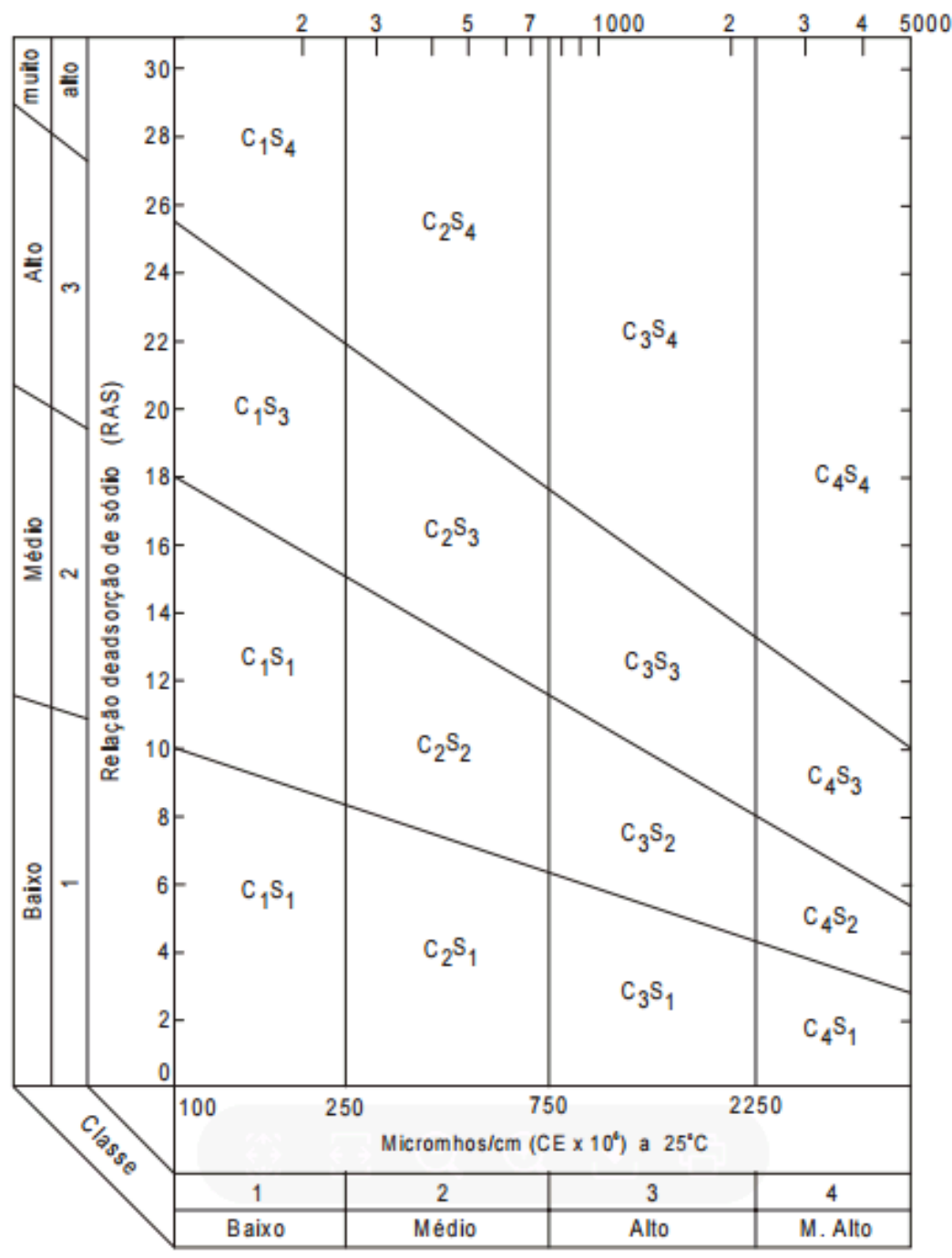

Fonte: CORDEIRO, G.G., 2001 


\subsection{Toxidade}

A toxidade é problema desenvolvido pelo acumulo de íons, que são absorvidos pelas raízes do vegetal e se fixam nas folhas dos mesmos por meio da transpiração. Essa concentração provoca efeitos tóxicos nas plantas, principalmente os íons cloro, boro e sódio, que normalmente se encontram nas águas de irrigação (SILVA, I. N. et al. 2011).

O íon de cloro provoca a degeneração em necrose nas bordas das folhas. Por ser ânion, é absolvido, por meio do método de irrigação por aspersão, pelas raízes e folhas das plantas, o que diminui a absorção do nitrogênio e fosforo pela mesma (AYERS, R. S.; WESTCOT, D. W, 1991).

Ainda segundo o mesmo autor, a toxidade do sódio está na concentração de cátion, observada principalmente pelo método de aspersão. Sua toxidade resulta em queimaduras nas folhas das plantas e na destruição da textura do solo, impedindo assim a absorção de outros nutrientes. A toxidade por boro está associada aos efeitos gerados sobre as folhas mais antigas da planta, aonde os bordos vão se amarelando e secando os tecidos da mesma. De um modo geral, a Tabela 3 demostra os critérios da qualidade da água destinados à irrigação (SILVA, I. N., 2011.).

Tabela 2: Classificação geral da água na irrigação.

\begin{tabular}{|c|c|c|c|c|}
\hline \multirow{2}{*}{ Problema Potencial } & \multirow{2}{*}{ Unidade } & \multicolumn{3}{|c|}{ Grau de restrição de uso } \\
\hline & & Nenhum & Moderado & Severo \\
\hline \multicolumn{5}{|l|}{ SALINIDADE } \\
\hline $\mathrm{CE}_{\mathrm{ai}}$ & $\mathrm{dS} / \mathrm{m}$ & $<0,7$ & 0,7 a 3,0 & $>3,0$ \\
\hline TSD & $\mathrm{mg} / \mathrm{L}$ & $<450$ & 450 a 2000 & $>2000$ \\
\hline \multicolumn{5}{|l|}{ INFILTRAÇÃO } \\
\hline $\mathrm{RAS}=0$ a $3 \quad$ e CE $=$ & $\mathrm{dS} / \mathrm{m}$ & $>0,7$ & $0,7-0,2$ & $<0,2$ \\
\hline $\mathrm{RAS}=3$ a $6 \quad$ e $C E=$ & $\mathrm{dS} / \mathrm{m}$ & $>1,2$ & $1,2-0,3$ & $<0,3$ \\
\hline $\mathrm{RAS}=6$ a 12 e CE $=$ & $\mathrm{dS} / \mathrm{m}$ & $>1,9$ & $1,9-0,5$ & $<0,5$ \\
\hline $\mathrm{RAS}=12$ a 20 e CE $=$ & $\mathrm{dS} / \mathrm{m}$ & $>2,9$ & $2,9-1,3$ & $<1,3$ \\
\hline $\mathrm{RAS}=20$ a 40 e $C E=$ & $\mathrm{dS} / \mathrm{m}$ & $>5,0$ & $5,0-2,9$ & $<2,9$ \\
\hline \multicolumn{5}{|l|}{ TOXIDADE } \\
\hline Sódio (Na+) & RAS & $<3,0$ & $3,0-9,0$ & $>9,0$ \\
\hline Irrigação por superfície & m.e./L & $<3,0$ & $3,0-9,0$ & $>9,0$ \\
\hline \multicolumn{5}{|l|}{ Irrigação por aspersão } \\
\hline \multicolumn{5}{|l|}{ Cloro (Cl) } \\
\hline Irrigação por superfície & m.e./L & $<4,0$ & $4,0-10,0$ & $>10,0$ \\
\hline Irrigação por aspersão & m.e./L & $<3,0$ & $>3,0$ & \\
\hline Boro (Bo) & m.e./L & $<0,7$ & 0,7 a 3,0 & $>3,0$ \\
\hline \multicolumn{5}{|l|}{ MISCELÂNEA } \\
\hline Nitrogênio $\left(\mathrm{NO}_{3-} \mathrm{N}\right)$ & $\mathrm{mg} / \mathrm{L}$ & $<5,0$ & 5,0 a 30,0 & $>3,0$ \\
\hline \multicolumn{5}{|l|}{ Bicarbonato $\left(\mathrm{HCO}_{3}{ }^{-}\right)$} \\
\hline IRRIGAÇÃO POR ASPERSÃO & & $<1,5$ & $1,5-8,5$ & $>8,5$ \\
\hline $\mathrm{pH}$ & \multicolumn{4}{|c|}{ Amplitude normal de 6,5 a 8,4 } \\
\hline
\end{tabular}

Fonte: SILVA, I. N., 2011. 


\section{CONCLUSÃO}

A partir dos estudos realizados, se verificou os cálculos específicos e os critérios de cada parâmetro aplicados na avaliação da titulação da água para uso da irrigação. Estes testes analisam a concentração de cada substância encontrada na água, bem como seus possíveis danos gerados na produtividade da planta cultivada e no solo a ser utilizado.

Sobre tudo estes parâmetros demostram a importância no planejamento e no monitoramento dos recursos a serem utilizados, para que se amenizem tais danos e prejuízos futuros ao produtor.

\section{AGRADECIMENTO}

Agradecemos a professora Dr. a Camila Pires Cremasco pela ajuda e orientação para que pudéssemos escrever este trabalho, e o XII Fórum Ambiental da Alta Paulista por conceder o espaço para publicação do mesmo.

\section{REFERÊNCIA}

ALMEIDA, Otávio Álvares de. Qualidade da água de irrigação. Dados eletrônicos. Cruz das Almas: Embrapa Mandioca e fruticultura, 2010. Disponível em: <http://www.cnpmf.embrapa.br/publicacoes/livro qualidade agua.pdf >. Acesso em 20/01/2016.

AYERS, R. S.; WESTCOT, D. W. A qualidade da água na agricultura. Campina Grande: UFPB, 1991. 218 p. (Tradução).

BERNARDO, Salassier. Manual de irrigação. 4. ed. Viçosa: UFV, Imprensa Universitária, 1987. 488 p.

BERNARDO, Salassier; SOARES, Antônio Alves; MANTOVANI, Everardo Chartuni. Manual de irrigação. 8. ed. Viçosa: UFV, 2006. 625 p

CORDEIRO, Gilberto Gomes. Qualidade de água para fins de irrigação (Conceitos básicos e práticas). Petrolina, PE: Embrapa Semi- Árido, 2001. 32 p.

MANTOVANI, Everardo Chartuni; BERNARDO, Salassier; PALARTTI, Luiz Fabiano . Irrigação: princípios e métodos. Viçosa: UFV, 2006. $328 \mathrm{p}$.

PAZ, Vital Pedro da Silva; TEODORO, Reges Eduardo Franco; MENDONÇA, Fernando Campos. Recursos hídricos, agricultura irrigada e meio ambiente. R. Bras. Eng. Agríc. Ambiental, Campina Grande, v.4, n.3, 2000. 465-473 p.

SILVA, Ítalo Nunes et al. Qualidade de água na irrigação. ACSA - Agropecuária Científica no Semi-Árido, v.07, n 03 julho/setembro 2011. 15 p.

SUAREZ, D.L. Relation between $\mathrm{pHc}$ and sodium adsorption ratio (SAR) and an alternative method of estimating SAR of soil or drainage waters. Soil Sci. Soc. Amer. J., n.45, p.469-75, 1981. 